\title{
Normative models of enhancer function
}

\author{
Rok Grah, ${ }^{*}$ Benjamin Zoller, ${ }^{\dagger}$ and Gašper Tkačik ${ }^{* \ddagger}$
}

\begin{abstract}
In prokaryotes, thermodynamic models of gene regulation provide a highly quantitative mapping from promoter sequences to gene expression levels that is compatible with in vivo and in vitro biophysical measurements. Such concordance has not been achieved for models of enhancer function in eukaryotes. In equilibrium models, it is difficult to reconcile the reported short transcription factor ( $\mathrm{TF}$ ) residence times on the DNA with the high specificity of regulation. In non-equilibrium models, progress is difficult due to an explosion in the number of parameters. Here, we navigate this complexity by looking for minimal non-equilibrium enhancer models that yield desired regulatory phenotypes: low TF residence time, high specificity and tunable cooperativity. We find that a single extra parameter, interpretable as the "linking rate" by which bound TFs interact with Mediator components, enables our models to escape equilibrium bounds and access optimal regulatory phenotypes, while remaining consistent with the reported phenomenology and simple enough to be inferred from upcoming experiments. We further find that high specificity in non-equilibrium models is in a tradeoff with gene expression noise, predicting bursty dynamics - an experimentallyobserved hallmark of eukaryotic transcription. By drastically reducing the vast parameter space to a much smaller subspace that optimally realizes biological function prior to inference from data, our normative approach holds promise for mathematical models in systems biology.
\end{abstract}

Keywords: transcriptional regulation | non-equilibrium models | noise in gene expression | enhancer function | Monod-Wyman-Changeux (MWC) model

An essential step in the control of eukaryotic gene ex- 50 pression is the interaction between transcription factors ${ }_{51}$ (TFs), various necessary co-factors, and TF binding sites ${ }_{2}$ (BSs) on the regulatory segments of DNA known as en- 53 hancers [1]. While we are far from having either a com- 54 plete parts list for this extraordinarily complex regula- 55 tory machine or an insight into the dynamical interac- 56 tions between its components, experimental observations 57 have established a number of constraints on its opera- 58 tion: (i) TFs individually only recognize short, $6-10 \mathrm{bp} 59$ long binding site motifs [2]; (ii) TF residence times on 60 the cognate binding sites can be as short as a few sec- 61 onds and only $2-3$ orders of magnitude longer than res- 62 idence times on non-specific DNA [3-5]; (iii) the order ${ }_{63}$ of arrival of TFs to their binding sites can affect gene 64 activation [4]; (iv) TFs do not activate transcription by 65 RNA polymerase directly, but interact first with various ${ }_{60}$ co-activators, essential amongst which is the Mediator ${ }_{67}$ complex; $(v)$ binding of multiple TFs is typically required ${ }_{68}$ within the same enhancer for its activation [6], which can ${ }_{69}$ lead to very precise downstream gene expression only in ${ }_{70}$ the presence of a specific combination of TF concentrations [7]; (vi) when activated, gene expression can be ${ }_{72}$ highly stochastic and bursty [8-10]; (vii) gene induction ${ }_{73}$ curves show varying degrees of steepness, suggesting tun- ${ }_{74}$ able amounts of cooperativity among TFs [11]. Here we ${ }_{75}$ look for biophysical models of enhancer function consis- ${ }_{76}$ tent with these observations.

Mathematical modeling of gene regulation traces its ${ }_{78}$

\footnotetext{
* Institute of Science and Technology Austria, AT-3400 ${ }^{81}$ Klosterneuburg, Austria

† Princeton University, NJ 08544 Princeton, USA; Institut Pas- 83 teur, FR-75015 Paris, France

$\ddagger$ Corresponding author. Email: gtkacik@ist.ac.at
}

origins to the paradigmatic examples of the $\lambda$ bacteriophage switch [12] and the lac operon [13]. In prokaryotes, biophysical models have proven very successful [14-16], assuming gene expression to be proportional to the fraction of time RNA polymerase is bound to the promoter in thermodynamic equilibrium; TFs modulate this fraction via steric or energetic interactions with the polymerase. Crucially, these models are very compact: they are fully specified by enumerating all bound configurations and energies of the TFs and the polymerase on the promoter. While some open questions remain [17-19], the thermodynamic framework has provided a quantitative explanation for combinatorial regulation, cooperativity, and regulation by DNA looping [20, 21], while remaining consistent with experiments that also probe the kinetic rates $[22,23]$.

No such consensus framework exists for eukaryotic transcriptional control. Limited specificity of individual TFs (i) is hard to reconcile with the high specificity of regulation $(v)$ and the suppression of regulatory crosstalk [24], suggesting non-equilibrium kineticproofreading schemes [25]. Likewise, short TF residence times (ii) and the importance of TF arrival ordering (iii) contradict the conceptual picture where stable enhanceosomes are assembled in equilibrium [4]. Kinetic schemes may be required to match the reported characteristics of bursty gene expression (vi) [26], or realize high cooperativity (vii) [27]. Thermodynamic models undisputedly have statistical power to predict expression from regulatory sequence even in eukaryotes [28], yet this does not resolve their biophysical inconsistencies or rule out nonequilibrium models. Unfortunately, mechanistically detailed non-equilibrium models entail an explosion in the complexity of the corresponding reaction schemes and the number of associated parameters: on the one hand, such models are intractable to infer from data, while on 
the other, it is difficult to understand which details are ars $_{13}$ essential for the emergence of regulatory function.

To deal with this complexity, we systematically sim-135 plify the space of enhancer models. We adopt the norma-136 tive approach, commonly encountered in the applications137 of optimality ideas in neuroscience and elsewhere [29-138 31]: we theoretically identify those models for which var-139 ious performance measures of gene regulation, which we140 call "regulatory phenotypes", are maximized. Such op-141 timal model classes are our candidates that could subse-142 quently be refined for particular biological systems and 143 confronted with data. Thus, rather than inferring a sin-144 gle model from experimental data or constructing a com-145 plex, molecularly-detailed model for some specific en-146 hancer, we find the simplest generalizations of the clas-147 sic equilibrium regulatory schemes, such as Hill-type [32 ${ }_{148}$ or Monod-Wyman-Changeux regulation [33-35], to non-149 equilibrium processes, which drastically improves their 150 regulatory performance while leaving the models simple151 to analyze, simulate, and fit to data.

\section{RESULTS}

\section{A. Model.}

Multiple lines of evidence suggest that eukaryotic tran- ${ }^{159}$ scription is a two-state process which switches between ${ }^{160}$ active (ON) and inactive (OFF) states, with rates depen ${ }^{161}$ dent on the transcription factor (TF) concentrations [36- ${ }^{-162}$ 38]. We sought to generalize classic regulatory schemes ${ }^{163}$ that can describe the balance between ON and OFF tran- ${ }^{164}$ scriptional states in equilibrium: a Hill-like scheme of ${ }^{165}$ "thermodynamic models" (discussed in SI Section 1.3), ${ }^{166}$ and a Monod-Wyman-Changeux-like (MWC) scheme in ${ }^{167}$ troduced below.

Figure 1A shows a schematic of the proposed functional enhancer model (SI Section 1.1, see also Fig S1) ${ }_{169}$ A complex of transcriptional co-factors that we refer to as a "Mediator" ${ }^{1}$ can interact with TFs that bind and unbind from their DNA binding sites with baseline rates ${ }^{170}$ $k_{+}$and $k_{-}$(Fig 1B.i). Mediator - and thus the whole en ${ }^{171}$ hancer - can switch between its functional ON/OFF states ${ }^{172}$ with baseline rates $\kappa_{+}$and $\kappa_{-}$(Fig 1B.ii). Enhancer $\mathbf{O N}^{173}$ state and TF bound state are both stabilized (by a factor ${ }^{174}$ $\alpha$ relative to baseline rates) when a bound TF establishes ${ }^{175}$ a "link" with the Mediator (Fig 1B.iii). The molecularr" identity of such links can remain unspecified: it could, for ${ }^{177}$ example, correspond to an enzymatic creation of chem- ${ }^{178}$ ical marks (e.g., methylation, phosphorylation) on the $\mathrm{e}^{179}$ TFs or Mediator proteins conditional on their physical ${ }^{180}$

${ }^{1}$ Our nomenclature is simply a shorthand for all co-factors necessary for eukaryotic transcriptional activation at an enhancer, 182 which can include proteins not strictly a part of the Mediator ${ }_{183}$ family. proximity or interaction. Crucially, the links can be established and removed in processes that can break detailed balance and are thus out of equilibrium. Here, we consider that a link is established at a rate $k_{\text {link }}$ between a bound $\mathrm{TF}$ and the Mediator complex; for simplicity, we assume that the links break when the TFs dissociate or upon the switch into OFF state (this assumption can be relaxed, see Fig S2).

An important thrust of our investigations will concern the role of limited specificity of individual TFs to recognize their cognate sequences on the DNA. If sequence specificity arises primarily through TF binding - a strong, but relatively unchallenged assumption (that can also be relaxed within our framework, see Fig S3) - then we should ask how likely it is for the Mediator complex to form and activate at specific sites contained within functional enhancers (with low off-rates characteristic of strong eukaryotic TF binding sites, $k_{-}^{\mathrm{S}}$ ) versus at random, non-specific sites on the DNA (with $\sim 2$ ordersof-magnitude higher individual TF off-rates, $k_{-}^{\mathrm{NS}}$ ) from which expression should not occur.

Given the number of TF binding sites $(n)$ and the various rate parameters $\left(k_{+}, k_{-}^{\mathrm{S} / \mathrm{NS}}, \kappa_{+}, \kappa_{-}, \alpha, k_{\text {link }}\right)$ the full state of the system - i.e., the probability to observe any number of bound and/or linked TFs jointly with the ON/OFF state of the enhancer - evolves according to a Chemical Master Equation (SI Section 1.1) that can be solved exactly [39-41] or simulated using the Stochastic Simulation Algorithm [42]. Importantly, we show analytically that our scheme reduces to the true equilibrium MWC model in the limit $k_{\text {link }} \rightarrow \infty$ : in this limit, there can be no distinction between a bound $\mathrm{TF}$ and a $\mathrm{TF}$ that is both bound and linked, and one can define a free energy $F$ that governs the probability of enhancer being ON, which in our model is equal to (a normalized) mean expression level, $E=P_{\mathrm{ON}}=(1+\exp (F))^{-1}$, with

$$
F=n \log \frac{1+c / K}{1+\alpha \cdot c / K}-L,
$$

where $K=k_{-} / k_{+}^{0}, k_{+}=k_{+}^{0} c$ (see also Fig 1 caption), and $L=\log \left(\kappa_{+} / \kappa_{-}\right)$. The $k_{\text {link }}$ parameter thus interpolates between the equilibrium limit in Eq (1), corresponding to a textbook MWC model, and various nonequilibrium (kinetic) schemes which we will explore next. A similar generalization with an equilibrium limit exists for thermodynamic Hill-type models, where, furthermore, $\alpha$ can be directly identified with cooperativity between DNA-bound TFs (see SI Section 1.3); we will see that this qualitative role of $\alpha$ will hold also for the MWC case.

\section{B. Regulatory phenotypes.}

How does the regulatory performance depend on the enhancer parameters and, in particular, on moving away from the equilibrium limit? To assess this question sys- 
A

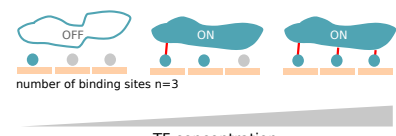

C

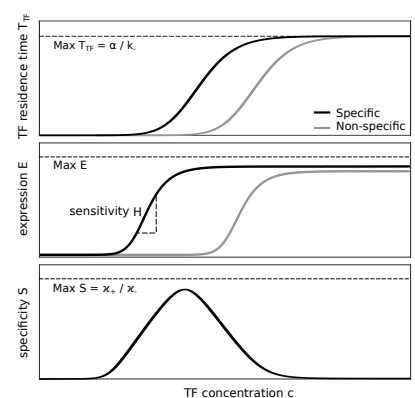

B (i) TF binding and unbinding

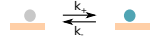

(ii) Mediator switching between ON and OFF state

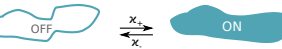

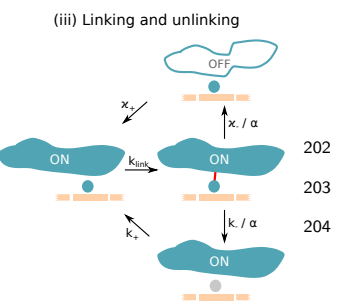

FIG. 1. A non-equilibrium MWC-like model of enhancer function. (A) Schematic representation of transcription factors (TFs; tael circles) interacting with binding ${ }^{206}$ sites (BSs, here $n=3$ orange slots) and the putative Mediator ${ }^{207}$ complex via links (red lines). The Mediator complex can be ${ }^{208}$ in two conformational states (OFF or ON), with the ON state en-209 abling productive transcription of the regulated gene. Increas-210 ing TF concentration, $c$, facilitates TF binding and the switch ${ }_{211}$ into $\mathrm{ON}$ state (left-to-right). (B) Key reactions and rates of the non-equilibrium model. TFs can bind with concentration- ${ }_{213}$ dependent on rate $\left(k_{+}=k_{+}^{0} c\right)$ and unbind with basal rate $k_{-}{ }^{213}$ that is in principle sequence dependent (i). The Mediator ${ }^{214}$ state switches between the conformational states with basal ${ }^{215}$ rates $\kappa_{+}$and $\kappa_{-}$(ii). Linking and unlinking of TFs to $\mathrm{Me}_{-}^{216}$ diator (iii) can move the system out of equilibrium: links ${ }^{217}$ are established with rate $k_{\text {link }}$, and the link stabilizes both ${ }^{218}$ $\mathrm{TF}$ residence and the $\mathrm{ON}$ state of the Mediator by a factor $\alpha^{219}$ per established link. (C) Regulatory phenotypes. Mean TF220 residence time, $T_{\mathrm{TF}}$, on specific sites in functional enhancers $\mathrm{S}_{221}$ (black) vs random site on the DNA (gray) increases with con- ${ }_{222}$ centration (top), as does mean expression, $E$ (the fraction of ${ }_{223}$ time the Mediator is ON; induction curve, middle, with sen- ${ }^{223}$ sitivity, $H$, defined at mid-point expression). Specificity, $S{ }^{224}$ is defined as the ratio of expression from the specific sites in ${ }^{225}$ the enhancer relative to the expression from random piece of ${ }^{226}$ DNA. tematically, we define a number of "regulatory pheno-23 types", enumerated in Table I and illustrated in Fig 1C.232 As a function of TF concentration, we compute: (i) $)_{233}$ individual $\mathrm{TF}$ residence time, $T_{\mathrm{TF}}$, on specific sites in 234 functional enhancers, as well as on random, non-specific 235 DNA, because these quantities have been experimen-236 tally reported in single-molecule experiments and pro-237 vide strong constraints on enhancer function; (ii) aver-238 age expression, $E$, for functional enhancers as well as239 random, non-specific DNA; we require $E$ to be in the240 middle $(\sim 0.5)$ of the wide range reported for functional 24 enhancers; (iii) sensitivity of the induction curve at half-242 maximal induction, $H$, an observable quantity often interpreted as a signature of cooperativity in equilibrium models; (iv) specificity, $S$, as the ratio between expression $E$ from functional enhancers vs from non-specific DNA, which should be as high as possible to prevent

\begin{tabular}{|l|c|c|c|}
\hline Phenotype & Symbol & Value & Ref \\
\hline \hline TF residence time (specific BS) & $T_{\mathrm{TF}}$ & $\sim 1-10 \mathrm{~s}$ & {$[3,43]$} \\
Expression (fraction of time ON) & $E$ & $0.01-0.9$ & {$[38,44,45]$} \\
Sensitivity (apparent Hill coef.) & $H$ & $1-10$ & {$[11]$} \\
Specificity & $S$ & - & - \\
Noise (std / mean protein exp.) & $N$ & $\sim 0.1-1$ & {$[46]$} \\
\hline
\end{tabular}

TABLE I. Regulatory phenotypes.

deleterious crosstalk or uncontrolled expression [24]; (v) expression noise, $N$, defined more precisely later, originating in stochastic enhancer ON/OFF switching. ${ }^{2}$

\section{Specificity, residence time, and expression.}

Figure 2A explores the relationship between three regulatory phenotypes for a MWC-like enhancer scheme of Fig 1A: the average $\mathrm{TF}$ residence time $\left(T_{\mathrm{TF}}\right)$, specificity $(S)$, and the average expression $(E)$, at fixed concentration $c_{0}$ of the TFs. Each point in this "phase diagram" corresponds to a particular enhancer model; points are accessible by varying $\alpha$ and $k_{\text {link }}$ (Fig 2B) and fall into a compact region that is bounded by intuitive, analyticallyderivable limits to specificity and the residence time. As $\alpha$ tends to large values, $S$ approaches 1 , as it must: once a TF-Mediator complex forms, large $\alpha$ will ensure it never dissociates and expression $E$ will tend to 1 (see also Fig 2D) irrespective of whether this occurred on a functional enhancer or a random piece of DNA - in this limit, all sequence discrimination ability is lost, yielding undesirable regulatory phenotypes. In contrast, the equilibrium ("EQ") MWC limit as $k_{\text {link }} \rightarrow \infty$ (Eq 1) is functional and, interestingly, corresponds to a non-monotonic curve in the phase diagram that lower-bounds the specificity of non-equilibrium ("NEQ") models accessible at finite values of $k_{\text {link }}$.

In a wide intermediate range of $\mathrm{TF}$ residence times, the full space of nonequilibrium MWC-like modelswhich we can exhaustively explore offers large, ordersof-magnitude improvements in specificity, essentially utilizing a stochastic variant of Hopfield's proofreading mechanism [25, 47]. This observation is generic, even though the precise values of $S$ depend on parameters that we explore below, and $S$ always remains bounded from above by $\kappa_{-} / \kappa_{+}$(in equilibrium, this is related to stochastic, thermal-fluctuation-driven Mediator transitions to ON state even in absence of bound TFs). At the same average $\mathrm{TF}$ residence time and $\mathrm{TF}$ concentration, the best non-equilibrium model (II in Fig 2) will suppress expression from non-cognate DNA by almost two orders-of-magnitude relative to the best equilibrium model (I). These findings remain qualitatively unchanged

\footnotetext{
${ }^{2}$ Protein noise levels in Table I are estimated from reported mRNA noise levels.
} 

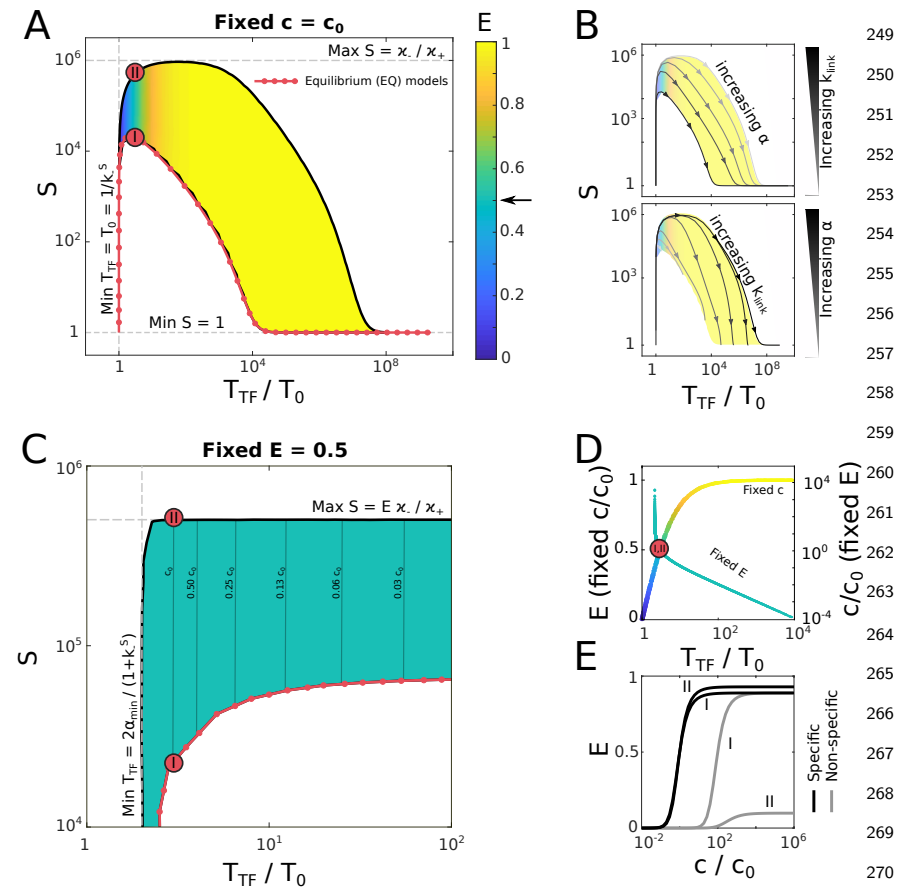

FIG. 2. Accessible space of regulatory phenotypes.272 (A) Specificity, $S$, mean $\mathrm{TF}$ residence time, $T_{\mathrm{TF}}$ (expressed ${ }_{273}$ in units in inverse off-rate for isolated TFs at their specific ${ }_{274}$ sites, $T_{0}=1 / k_{-}^{\mathrm{S}}$ ), and average expression, $E$ (color), for ${ }_{275}$ MWC-like models with $n=3 \mathrm{TF}$ binding sites, obtained by ${ }_{276}$ varying $\alpha$ and $k_{\text {link }}$ at fixed TF concentration, $c_{0}$. Equilibrium models fall onto the red line; two models with equal $\mathrm{TF}^{277}$ residence times, I (EQ) and II (NEQ), are marked for com- $^{278}$ parison. Dashed gray lines show analytically-derived bounds. ${ }^{279}$ (B) Phase space of regulatory phenotypes is accessed by vary-280 ing $\alpha$ at fixed values of $k_{\text {link }}$ (grayscale; top) or varying $k_{\text {link }}{ }^{281}$ at fixed values of $\alpha$ (grayscale; bottom). (C) As in (A), but282 the $\mathrm{TF}$ concentration at each point in the phase space is ad-283 justed to hold average expression fixed at $E=0.5$ (green $_{284}$ color). Plotted is a smaller region of phase space of inter- ${ }_{285}$ est; nearly vertical thin lines are equi-concentration contours (Fig S6). (D) All models in the phase diagrams in (A) and (C) approximately collapse onto nearly one-dimensional manifolds ("fixed c", left axis, for (A); "fixed E", right axis, for ${ }^{286}$ (C)) when plotted as a function of mean TF residence time, $T_{\mathrm{TF}}$, supporting the choice of this variable as a biologically- ${ }_{287}$ relevant observable. Color on the manifold corresponds to ${ }_{288}$ mean expression $E$ using the colormap of (A). Vertical scales ${ }_{289}$ are chosen so that models I and II coincide. (E) Induction ${ }^{289}$ curves of equilibrium model $\mathrm{I}$ and non-equilibrium model $\mathrm{II}^{290}$ for expression from functional enhancer that contains specific ${ }^{291}$ sites (basal TF off-rate $k_{-}^{S}$; black curves) versus expression ${ }^{292}$ from random DNA containing non-specific sites (basal $\mathrm{TF}^{293}$ off-rate $k_{-}^{\mathrm{NS}}=10^{2} k_{-}^{\mathrm{S}}$ here; gray curves).

for enhancers with larger number of binding sites $\left(\sec _{298}^{297}\right.$ Fig S4).

A comparison of various enhancer operating regimes is 300 perhaps biologically more relevant at fixed mean expres-301 sion, allowing the TF concentration to adjust accord-302 ingly under cells' own control, as shown in Fig 2C for ${ }_{30}$
$E=0.5$. As TF residence time lengthens with increasing $\alpha$, TFs and the Mediator establish more stable complexes on the DNA and lower concentrations are needed for all models to reach the desired expression $E$ (see also Fig 2D). Nevertheless, the ability of $\alpha$ to increase the specificity in equilibrium models is limited and saturates at a value substantially below the specificity reachable in nonequilibrium models at much smaller $\mathrm{TF}$ residence times. The observations of Figs 2A, C underscore an important, yet often overlooked, point: the ability to induce at low TF concentration (that is, high affinity) achieved through "cooperative interactions" at high $\alpha$ either has a detrimental, or, at best, a marginally beneficial effect for the ability to discriminate between cognate and random DNA sites (that is, high specificity) in equilibrium [24].

Figure 2E shows induction curves for expression from functional enhancers containing specific sites and from random DNA sites, for equilibrium (I) and nonequilibrium (II) models. Both yield essentially indistinguishable induction curves for expression from a functional enhancer (which is true generically across our phase diagram, see Fig S5), suggesting that it would be difficult to discriminate between the models based on induction curve measurements. In sharp contrast, the behavior of the two models is qualitatively different at non-specific DNA: with sufficiently high TF concentration (e.g., in an over-expression experiment), the EQ model I will fully induce even from random DNA as its binding sites get saturated by TFs; on the contrary, the nonequilibrium (NEQ) model II will start inducing at much higher $c$, and will never do so fully due to its proofreading capability. Thus, given the relatively weak individual TF preference for cognate vs non-cognate DNA, one should look at the collective response of the gene expression machinery to mutated or random enhancer sequences for signatures of equilibrium vs non-equilibrium proofreading behavior.

\section{Sensitivity.}

Intuitively, sensitivity $H$ measures the "steepness" of the induction curve. More precisely, $H$ is proportional to the logartihmic derivative of the expression with log concentration at the point of half-maximal expression, so that for Hill-like functions, $E(c)=c^{h} /\left(c^{h}+K^{h}\right)$, it corresponds exactly to the Hill coefficient, $H=h$. Figure $3 \mathrm{~A}$ shows that $H$ increases monotonically with $T_{\mathrm{TF}}$ (and thus with $\alpha$, cf. Fig 2B), indicating that more stable TF-Mediator complexes indeed lead to higher apparent cooperativity, which is always upper-bounded by the number of TF binding sites in the enhancer, $n$. The highly-cooperative "enhanceosome" concept [48] would, in our framework, correspond to an equilibrium limit with very high $\alpha$, and thus $H \sim n$; yet the analysis above predicts vanishingly small specificity increases as this limit is approached. In contrast, we observe that the point at which the specificity advantage of nonequi- 
A
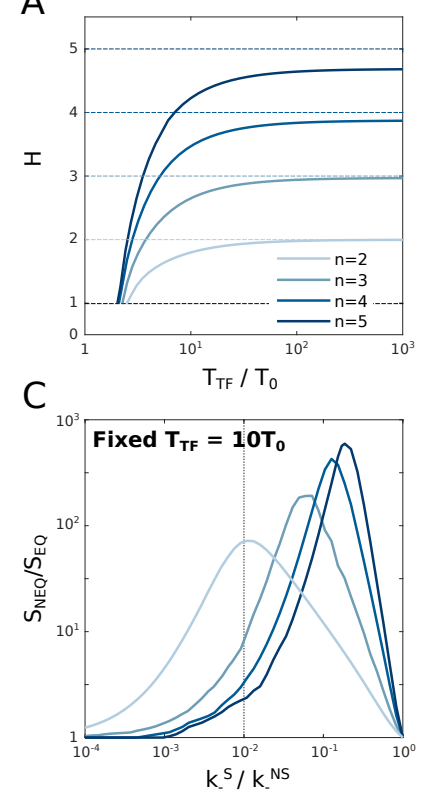

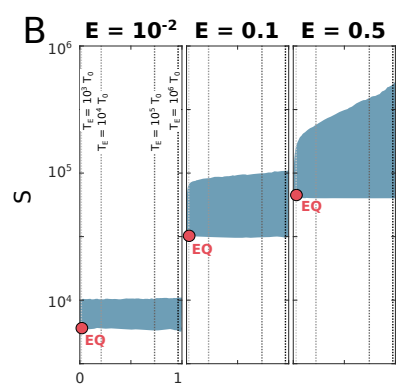

Expression noise $\Phi=N^{2} E /(1-E)^{324}$

$\mathrm{D}$

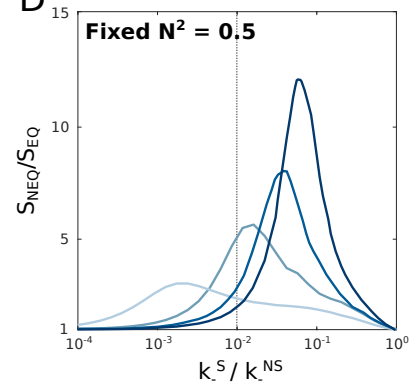
318

FIG. 3. Limits to sensitivity and specificity. (A) Sensi- ${ }^{336}$ tivity (apparent Hill coefficient) $H$ of enhancer models in the ${ }^{337}$ phase diagram of Fig $2 \mathrm{C}$, at fixed mean expression, $E=0.5 .338$ All models collapse onto the manifolds shown for different ${ }_{339}$ number of TF binding sites, $n$. (B) Phase diagram of en- ${ }_{340}$ hancer models for three different values of mean expression,,$_{341}$ $E$ (columns), shows specificity $S$ and fraction of variance in enhancer switching propagated to expression noise (see text). Compact blue region for each $E$ shows all MWC-like models ${ }^{343}$ with $n=3$ binding sites accessible by varying $\alpha$ and $k_{\text {link }} ;^{344}$ equilibrium model ("EQ") with lowest noise is shown as a red"345 dot. Increase in noise is monotonically related to increase in 346 enhancer correlation time, $T_{E}$, marked with dashed vertical ${ }_{347}$ lines. Largest specificity increases over EQ models occur at t $_{348}$ high $T_{E}$ and thus high noise (upper right corner of the blue ${ }_{349}$ region). (C) Maximal gain in enhancer specificity for nonequilibrium vs equilibrium models for different $n$ (legend as in A), as a function of the intrinsic specificity of individual ${ }^{35}$ $\mathrm{TF}$ binding sites, $k_{-}^{\mathrm{S}} / k_{-}^{\mathrm{NS}}$. Expression is fixed to $E=0.5^{352}$ and mean TF residence time to $T_{\mathrm{TF}} / T_{0}=10$. Typical value ${ }^{353}$ $k_{-}^{\mathrm{S}} / k_{-}^{\mathrm{NS}}=10^{-2}$ used in Fig 2 and panels $\mathrm{A}, \mathrm{B}$ is shown in ${ }^{354}$ vertical dashed line. (D) Same as in (C), but with the com- ${ }^{355}$ parison at fixed gene expression noise, $N^{2}=0.5$. librium models is maximized, i.e., where $S_{\mathrm{NEQ}} / S_{\mathrm{EQ}}$ is $_{359}$ largest, occurs far away from $H=n$, at much lower $H_{360}$ values (Fig S8). If high specificity is biologically favored, ${ }_{361}$ we should therefore not expect the "number of known ${ }_{362}$ binding sites" to equal the "measured Hill coefficient of ${ }_{363}$ the induction curve" for well-functioning eukaryotic tran- ${ }_{364}$ scriptional schemes, even on theoretical grounds.

E. Noise.

Lastly, we turn our attention to gene expression noise. 370 All stochastic two-state models have a steady state bino-371 mial variance of $\sigma_{E}^{2}=E(1-E)$ in enhancer state, where
$E$ is the probability of the enhancer to be ON. When ON, transcripts are made and subsequently translated into protein, which typically has a slow lifetime, $T_{P}$, on the order of at least a few hours. Random fluctuations in enhancer state will cause random steady-state fluctuations in protein copy number around the average, $P$; these fluctuations can be quantified by noise, $N=\sigma_{P} / P$. While there can be other contributions to noise (e.g., birthdeath fluctuations due to protein production and degradation), we focus here solely on the effects of ON/OFF switching, since only these effects depend on the enhancer architecture [30].

How is noise in gene expression, $N$, related to the binomial variance, $\sigma_{E}$ ? Based on simple noise propagation arguments $[49,50]$, fractional variance in protein should be equal to fractional variance in enhancer state times the noise filtering that depends on the timescales of enhancer switching, $T_{E}$, and protein lifetime, $T_{P}$ (here we assume $T_{P}=10$ hours), so that $N^{2}=\left(\sigma_{P} / P\right)^{2} \sim$ $\left(\sigma_{E} / E\right)^{2} \cdot T_{E} /\left(T_{E}+T_{P}\right)$ (see SI Section 1.5 for exact derivation). Thus, if enhancer switches much faster than the protein lifetime, $T_{E} \ll T_{P}$, protein dynamics almost entirely averages out the enhancer state fluctuations. Since all enhancer models have the same binomial variance, the gene expression noise in various models will be entirely determined by the mean expression, $E$, and the correlation time, $T_{E}$, both of which we can compute analytically for any combination of enhancer model parameters in the phase diagram of Fig 2.

Figure 3B shows the phase diagram of accessible MWC-like regulatory phenotypes for the specificity $(S)$, mean expression $(E)$ and fraction of enhancer switching noise that propagates to gene expression, $T_{E} /\left(T_{E}+T_{P}\right)$, found by varying $\alpha$ and $k_{\text {link }}$. As in Fig 2, equilibrium models ("EQ") have the lowest specificity $S$, but also lowest correlation time $T_{E}$ and thus lowest noise, regardless of the average expression, $E$. There exist NEQ models that achieve higher specificity at a small increase in noise, but the highest specificity increases always come handin-hand with a substantial lengthening of the correlation times in enhancer state fluctuations, and thus with the inevitable increase in noise.

To better elucidate the tradeoffs and limits to specificity in non-equilibrium vs equilibrium models, we next explore how enhancer specificity gains depend on the ability of individual TFs to discriminate cognate binding sites from random DNA in Fig 3C. If individual TFs permit very strong discrimination $\left(k_{-}^{\mathrm{S}} / k_{-}^{\mathrm{NS}}<10^{-4}\right.$; prokaryotic TF regime), NEQ models at fixed individual TF residence times, $T_{\mathrm{TF}}$, do not offer appreciable specificity increases in the collective enhancer response; in contrast, for the range around $k_{-}^{\mathrm{S}} / k_{-}^{\mathrm{NS}} \sim 10^{-2}$ typically reported for eukaryotic TFs, the specificity increase ranges from ten to thousand-fold, with the peak depending on the number of TF binding sites, $n$, as well as baseline Mediator specificity limit, $\kappa_{-} / \kappa_{+}$(as this increases, the peak specificity gain is higher and moves towards lower $k_{-}^{\mathrm{S}} / k_{-}^{\mathrm{NS}}$, see Fig S9). If, instead of fixing $k_{-}^{\mathrm{S}} / k_{-}^{\mathrm{NS}}=10^{-2}$ 
as we have done until now, we pick this ratio to maxi-428 mize the specificity gain $\left(S_{\mathrm{NEQ}} / S_{\mathrm{EQ}}\right)$ and again explore 429 the noise-specificity tradeoff as in Fig 3B, we find that 430 the extreme specificity gains are only possible when cor-43. relation times, $T_{E}$ diverge (see Fig S10), implying high ${ }_{432}$ noise.

These observations are summarized in Fig 3D, showing 434 the specificity gain of NEQ models relative to EQ models, 435 if the comparison is made at fixed noise level rather than ${ }^{436}$ at fixed individual TF residence time as in Fig 3C. Speci-437 ficity gains are limited to roughly ten-fold even when, as438 we do here, we systematically search for best NEQ mod-439 els through the complete phase diagram in Fig 2C. The440 specificity-noise tradeoff thus appears unavoidable.

\section{F. Experimentally observable signatures of enhancer function.}

To illustrate how the proposed nonequilibrium (NEQ) ${ }^{447}$ MWC-like scheme could function in practice, we simu- ${ }^{448}$ lated it explicitly and compared it to an equilibrium (EQ) $)^{449}$ scheme with the same mean TF residence time in Fig $4 .{ }^{450}$ The two enhancers, composed of $n=5 \mathrm{TF}$ binding sites, ${ }^{451}$ respond to a simulated protocol where the TF concen ${ }^{452}$ tration is first switched from a minimal value that drives ${ }^{453}$ essentially no expression to a high value giving rise to ${ }^{454}$ $E=0.5$, and after a long stationary period, the con- ${ }^{455}$ centration is switched back to the low value. Figure $4 \mathrm{~A}^{456}$ shows the occupancy of the binding sites and the func- ${ }^{457}$ tional ON/OFF state of the enhancer. Even though the ${ }^{458}$ two models share the same TF mean residence time and $d^{459}$ nearly indistinguishable induction curves (with $H \sim 2.7$ ), ${ }^{460}$ their collective behaviors are markedly different: the $\mathrm{EQ}^{461}$ scheme appears to have significantly faster $\mathrm{TF}$ binding ${ }^{462}$ / unbinding as well as Mediator switching dynamics, ${ }^{463}$ whereas NEQ scheme undergoes long, "bursty" periods ${ }^{464}$ of sustained enhancer activation and TF binding that ${ }^{465}$ are punctuated by OFF periods. If the typical residence ${ }^{466}$ time of an isolated TF on its specific site were $T_{0}=1 \mathrm{~s}{ }^{467}$ NEQ enhancer could stay active even for hour-long periods $\left(\sim 10^{4} \mathrm{~s}\right)$, just somewhat shorter than the protein lifetime $\left(\sim 4 \cdot 10^{4} \mathrm{~s}\right)$. Such enhancer-associated stable ${ }^{468}$ mediator clusters are consistent with recent experimental reports [51, 52].

The detailed steady-state behavior at high TF concen-470 tration is analyzed in Fig 4B. Consistent with our the-471 oretical expectations, the NEQ scheme enables ten-fold 442 higher specificity but at the cost of substantial noise in 473 gene expression $(N \sim 0.42)$ due to strong transcriptional 474 bursting. High noise is a direct consequence of the much 475 longer correlation time of enhancer fluctuations, $T_{E}$, for 476 the NEQ scheme, seen in Fig 4A. Interestingly, the mean 477 residence time of the enhancer $\mathrm{ON}$ state, $T_{M}$, is nearly478 unchanged between the EQ and NEQ scheme at $\sim 100_{479}$ s: but here, the mean turns to be a highly misleading ${ }_{480}$ statistic, as revealed by an in-depth exploration of the 481 full probability density function. The NEQ scheme has a482 long tail of extended $\mathrm{ON}$ events interspersed with an excess of extremely short OFF events (due to high $\kappa_{-}$rate necessary for high specificity) relative to the EQ scheme (which, itself, does not deviate strongly from an exponential density function with a matched mean). The behavior of such an enhancer is highly cooperative even though the sensitivity $(H)$ is not maximal: when the enhancer is ON, with very high probability all TFs are bound, and when OFF, often 4 out of 5 TFs are bound - yet the enhancer is not activated. In sum, a well-functioning nonequilibrium regulatory apparatus with its Mediator complex makes many short-lived attempts to switch ON, but only commits to a long, productive ON interval rarely and collectively, after insuring that activation is happening due to a sequence of valid molecular recognition events between several TFs and their cognate binding sites in a functional enhancer.

Transient behavior after a TF concentration change is analyzed in Fig 4C. The mean response time of the two models to the concentration change is governed by the correlation time of the enhancer state, $T_{E}$, and is thus much slower for NEQ vs EQ models; but since the protein lifetime is even longer, the mean protein levels adjust equally quickly in the equilibrium and nonequilibrium cases. This suggests that the dynamics of the mean protein level is unlikely to discriminate between EQ and NEQ models. In contrast, live imaging of the nascent mRNA could put constraints on $T_{E}$ [1]. In that case, the filtering time scale is the elongation time, typically on the order of a few minutes, while the reported transcriptional response times - and thus estimates of $T_{E}$-would range from minutes to $1-2$ hours $[9,26]$.

Steady-state noise levels at high induction, as reported already, are considerably higher for the NEQ model due to transcriptional bursting; an intriguing further suggestion of our analyses is a long transient in the noise levels upon a high-to-low TF concentration switch, which finally settles to a high fractional noise level (here, $N \sim$ 1.6) even at very low induction, due to sporadic transcriptional bursts.

\section{DISCUSSION}

In this paper, we took a normative approach to address the complexity of eukaryotic gene regulatory schemes. We proposed a minimal extension to a wellknown Monod-Wyman-Changeux model that can be applied to the switching between the active and inactive states of an enhancer. The one-parameter extension is kinetic and accesses nonequilibrium system behaviors. We analyzed the parameter space of the resulting model and visualized the phase diagram of "regulatory phenotypes", quantities that are either experimentally constrained (such as mean expression, mean TF residence time, sensitivity), are likely to be optimized by evolutionary pressures (such as noise and specificity), or both. This allowed us to recognize and understand biophysical 
A

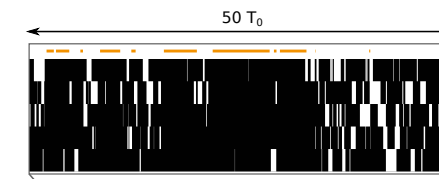

ITF bound

ITF unbound

- ON state
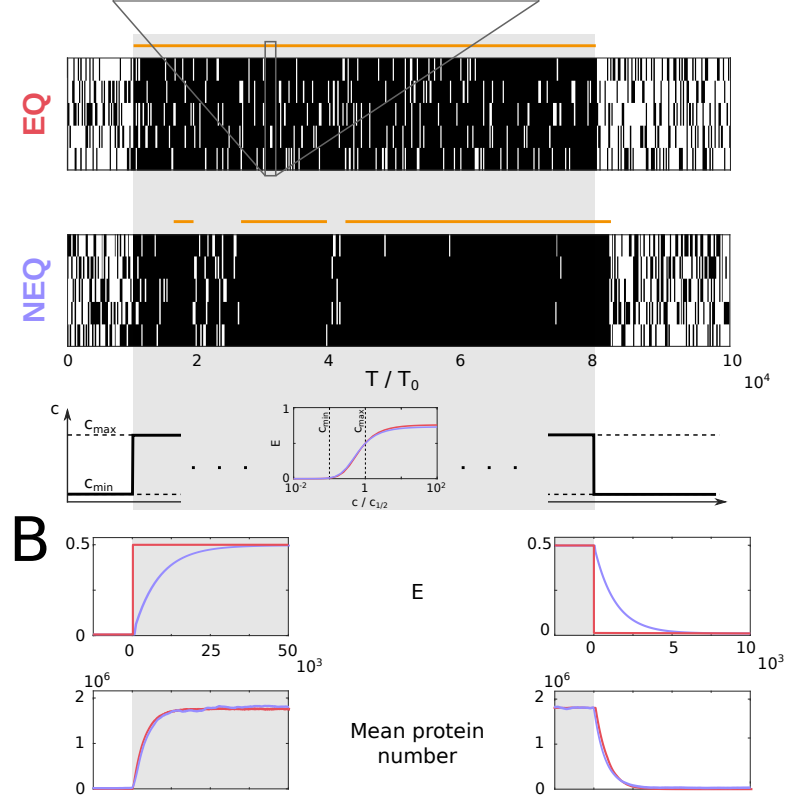

Mean protein number

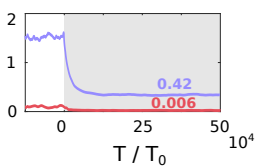

Protein

noise $\mathrm{N}$
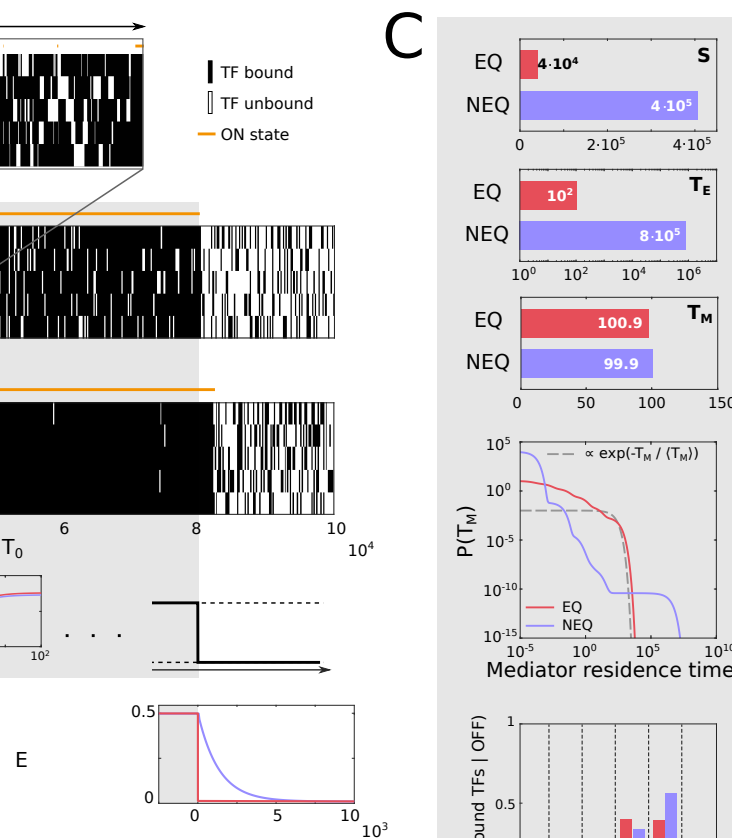

EQ
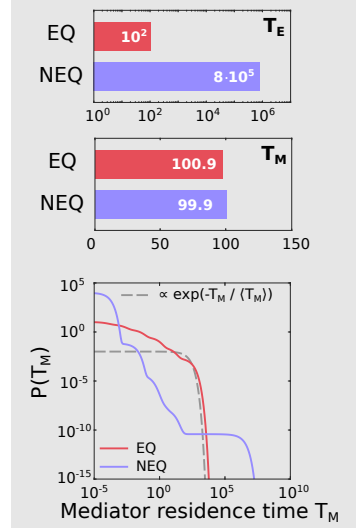

equilibrium (EQ) and a nonequilibrium (NEQ) enhancer model with $n=5 \mathrm{TF}$ binding sites, responding to a TF concentration step (bottom-most panel). Average TF residence times are the matched between EQ and NEQ models at $2.1 T_{0}, T_{0}=1 / k_{-}^{\mathrm{S}}=1$ $\mathrm{s}$, and both induction curves (scaled for half-maximal concentration) are identical, with sensitivity $H \approx 2.7$. When TF concentration is high, expression is fixed at $E=0.5$. Parameters for NEQ model: $\alpha=127, k_{\text {link }}=2, c_{\max }=0.065$; for EQ model: $k_{\text {link }} \rightarrow \infty, \alpha=19.8, c_{\max }=0.037$. Rasters show the occupancy of TF binding sites; orange line above shows the enhancer ON/OFF state; zoom-in for EQ model is necessary due to its fast dynamics. (B) Regulatory phenotypes for EQ and NEQ models during steady-state epoch (gray in A). Specificity $(S)$ and enhancer state correlation time $\left(T_{E}\right)$ are higher for the NEQ model; the Mediator mean ON residence time, $T_{M}$, is the same between the models, but the probability density function reveals a long tail in the NEQ scheme, and a nearly exponential distribution for the EQ scheme. Last two panels show the TF occupancy histogram during high TF concentration interval, conditional on the enhancer being OFF or ON. (C) Transient behavior of the mean enhancer state $(E)$, mean protein number $(P$; assuming deterministic production/degradation protein dynamics given enhancer state), and gene expression noise, $N=\sigma_{P} / P$, for the NEQ and EQ models, upon a TF concentration low-to-high switch (left column) and high-to-low switch (right column). Traces shown are computed as averages over 1000 stochastic simulation replicates.

limits and trade-offs, and to identify the optimal operat-495 ing regime of the proposed enhancer model that is con-496 sistent with current observations, as we summarize next.497

Our analyses suggest the following: (i) individual $\mathrm{TFs}_{499}$ are limited in their ability to discriminate specific from $_{500}$ random sites, $k_{-}^{\mathrm{S}} / k_{-}^{\mathrm{NS}} \sim 10^{-2}$, so high specificity must ${ }_{501}$ be a collective enhancer effect in the proofreading regime ${ }_{502}$ where $k_{\text {link }} \sim k_{-}^{\mathrm{S}}$; (ii) mean TF residence times in an en- ${ }_{503}$ hancer are not much higher than the typical TF residence ${ }_{504}$ time at an isolated specific site, $T_{\mathrm{TF}} / T_{0} \lesssim 10$, enabling ${ }_{505}$ rapid turnover of bound TFs on the $1-10$ s timescale; 500 (iii) typical sensitivities are much lower than the total number of TF binding sites, yielding a reasonable specificity/noise balance at $H \sim n / 2$ (Fig S7,S8); (iv) Mediator basal rates should maximize $\kappa_{-} / \kappa_{+}$, i.e., mediator switches OFF essentially instantaneously if not stabilized by linked TFs; ( $v$ TF concentrations required to activate the enhancer in this regime are substantially higher than expected for the equivalent but highly cooperative enhanceosome (at higher $\alpha$ ); (vi) optimal nonequilibrium models achieve order-of-magnitude improvements in $S$ relative to matched equilibrium models - thereby avoiding crosstalk and spurious gene expression - by suppressing induction from non-cognate (random) DNA, while 
induction curves from functional enhancers bear no clear 556 signatures of non-equilibrium operation; (vii) to permit557 large increases in specificity $S$, enhancer state fluctua-558 tions will develop long timescale correlations, $T_{E} \gg T_{\mathrm{T} F 559}$ (but still be bounded by the protein lifetime, $T_{E} \lesssim T_{P}$ t0560 enable noise averaging), leading to substantial observed ${ }_{561}$ noise levels; (viii) the enhancer ON residence time dis-562 tribution will be non-exponential, with excess probabil-563 ity for very long-lived events, during which an enhancer 564 could trigger a transcriptional burst following an interac-565 tion with the promoter; $(i x)$ in our model, long correla-566 tion time, $T_{E}$, in steady state also implies long (minutes567 to hours) response times when TF concentration change,568 which would be observable with live imaging on the tran-569 scriptional, but likely not protein-concentration, level. 570

We find it intriguing that a single-parameter exten- ${ }^{571}$ sion of a classic equilibrium model led to such richness ${ }^{572}$ of observed behaviors, and to a suggestion that the opti- ${ }^{573}$ mal operating regime is very different from regulation $a^{574}$ equilibrium. Central to this qualitative change is the fact $t^{575}$ that long fluctuation and response timescales of enhancer ${ }^{576}$ activation appear necessary to achieve high specificity of ${ }^{57}$ regulation through proofreading. Such long timescales ${ }^{578}$ are not inconsistent with our current knowledge. In- ${ }^{579}$ deed, some developmental enhancers form active clus- ${ }^{50}$ ters (super-enhancers) that are rather long-lived (order ${ }^{581}$ of minute to hours), perhaps precisely because develop- ${ }^{582}$ mental events need to be guided with extraordinary pre- ${ }^{53}$ cision $[52,53]$.

A strong objection to our model could be that it is ${ }^{585}$ too simple: after all, we neglected many structural $a d_{587}$ molecular details, many of which we may not even know $_{588}$ yet. This is certainly true and was done, in part, on pur- ${ }_{580}$ pose, to permit exhaustive analysis across the complete ${ }_{590}$ parameter space. Such understanding would have been ${ }_{591}$ impossible if we explored much richer models or were con- $_{592}$ cerned with quantitative fitting to a particular dataset. ${ }_{593}$ These are clearly the next steps, to which we contribute ${ }_{594}$ by highlighting the functional importance of breaking the ${ }_{595}$ equilibrium link between TF binding and enhancer acti- ${ }_{596}$ vation state. Since our model is fully probabilistic, spe- $^{-597}$ cializing it for a particular experimental setup, e.g., live transcriptional imaging, and doing rigorous inference is technically tractable, but beyond the scope of this paper. ${ }_{598}$

Perhaps a key simplification of our model is the link between enhancer / Mediator ON state and transcrip-599 tional activity. We assumed that expression is propor-600 tional to the probability of enhancer state to be ON, yet601 the enhancer-promoter interaction itself is a matter of vi-602 brant current experimentation and modeling $[10,51,54-$ $56]$. For example, long-lived activated enhancers that we predict could interact with promoters only intermittently to trigger transcriptional bursts, as suggested by the "dynamic kissing model" [52], which could substantially impact the experimentally-observable quantitative noise signatures of enhancer function at the transcriptional level. Whatever the true nature of enhancer-promoter interactions might be, however, they are unlikely to be able to remove excess enhancer switching noise, due to its slow timescale, suggesting that the tradeoffs that we identify should hold generically.

One could also question whether the importance we ascribed to high specificity is really warranted. Evolutionarily, regulatory crosstalk due to lower specificity helps networks evolve during transient bouts of adaptation, even though it could be ultimately selected against [57]. Mechanistically, molecular mechanisms such as chromatin modification or the regulated 3D structure of DNA decrease the number of possible non-cognate targets that could trigger erroneous gene expression [58, 59], and thus alleviate the need for the high specificity of the transcriptional control. Empirically, there is ample evidence for abortive or non-sensical transcriptional activity [60, 61], whose products could be dealt with downstream or simply ignored by the cell. Yet it is also clear that regulatory specificity must be a collective effect, as individual TFs bind pervasively across DNA even in non-regulatory regions [62], and self-consistent arguments suggest that in absence of non-equilibrium mechanisms, crosstalk could be overwhelming in eukaryotes [24]. It is also possible that real enhancers are very diverse with large variation along the specificity axis, thereby navigating the noisespecificity tradeoff as appropriate given the biological context. Where some erroneous induction can be tolerated, expression could be quicker, less noisy, and closer to equilibrium. In contrast, where tight control is needed, enhancers could take a substantial amount of time to commit to expression correctly, perhaps benefitting additionally from extra time-averaging that could further reduce the Berg-Purcell-type noise intrinsic to TF concentration sensing [50,63-65].

\section{ACKNOWLEDGMENTS}

GT acknowledges the support of the Human Frontiers Science Program RGP0034/2018. RG was supported by the Austrian Academy of Sciences DOC fellowship. RG thanks S. Avvakumov for helpful discussions.
[1] Antoine Coulon, Carson C. Chow, Robert H. Singer, and608 Daniel R. Larson. Eukaryotic transcriptional dynam-609 ics: from single molecules to cell populations. Nature610 Reviews Genetics, 14(8):572-584, August 2013. ISSN 611 1471-0056, 1471-0064. doi:10.1038/nrg3484. URL http: 612 //www. nature.com/articles/nrg3484.

[2] Zeba Wunderlich and Leonid A Mirny. Different gene regulation strategies revealed by analysis of binding motifs. Trends in genetics, 25(10):434-440, 2009.

[3] J Christof M Gebhardt, David M Suter, Rahul Roy, 
Ziqing W Zhao, Alec R Chapman, Srinjan Basu, Tom677 Maniatis, and X Sunney Xie. Single-molecule imaging of 678 transcription factor binding to DNA in live mammalian679 cells. Nature Methods, 10(5):421-426, May 2013. ISSN680 1548-7091, 1548-7105. doi:10.1038/nmeth.2411. URL681 http://www .nature.com/articles/nmeth.2411.

[4] Jiji Chen, Zhengjian Zhang, Li Li, Bi-Chang Chen, An-683 drey Revyakin, Bassam Hajj, Wesley Legant, Maxime684 Dahan, Timothe Lionnet, Eric Betzig, Robert Tjian,685 and Zhe Liu. Single-Molecule Dynamics of Enhanceo-686 some Assembly in Embryonic Stem Cells. Cell, 156687 (6):1274-1285, March 2014. ISSN 00928674. doi:688 10.1016/j.cell.2014.01.062. URL http://linkinghub. 689 elsevier.com/retrieve/pii/S0092867414001974.

[5] Colin Thomas, Yingbiao Ji, Chao Wu, Haily Datz,691 Cody Boyle, Brett MacLeod, Shri Patel, Michelle Am-692 pofo, Michelle Currie, Jonathan Harbin, Kate Pechenk-693 ina, Niraj Lodhi, Sarah J. Johnson, and Alexei V.694 Tulin. Hit and run versus long-term activation of PARP-695 1 by its different domains fine-tunes nuclear processes.696 Proceedings of the National Academy of Sciences, page697 201901183, April 2019. ISSN 0027-8424, 1091-6490. doi:698 10.1073/pnas.1901183116. URL http://www .pnas.org/699 lookup/doi/10.1073/pnas.1901183116.

[6] Daria Shlyueva, Gerald Stampfel, and Alexander Stark.701 Transcriptional enhancers: from properties to genome-702 wide predictions. Nature Reviews Genetics, 15(4):272,703 2014.

[7] Mariela D. Petkova, Gaper Tkaik, William Bialek, Eric F.705 Wieschaus, and Thomas Gregor. Optimal Decoding 706 of Cellular Identities in a Genetic Network. Cell, 176 707 (4):844-855.e15, February 2019. ISSN 00928674. doi: 708 10.1016/j.cell.2019.01.007. URL https://linkinghub. ${ }_{709}$ elsevier.com/retrieve/pii/S0092867419300406. $\quad{ }_{710}$

[8] Damien Nicolas, Benjamin Zoller, David M. Suter,711 and Felix Naef. Modulation of transcriptional burst 712 frequency by histone acetylation. Proceedings of $f_{713}$ the National Academy of Sciences, page 201722330,,$_{114}$ June 2018. ISSN 0027-8424, 1091-6490. doi $:_{715}$ 10.1073/pnas.1722330115. URL http://www . pnas.org/716 lookup/doi/10.1073/pnas.1722330115.

[9] N. Molina, D. M. Suter, R. Cannavo, B. Zoller, I. Gotic,, 718 and F. Naef. Stimulus-induced modulation of transcrip- 719 tional bursting in a single mammalian gene. Proceed-720 ings of the National Academy of Sciences, 110(51):20563- ${ }_{721}$ 20568, December 2013. ISSN 0027-8424, 1091-6490. doi: $:_{722}$ 10.1073/pnas.1312310110. URL http://www.pnas.org/723 cgi/doi/10.1073/pnas.1312310110.

[10] CarolineR. Bartman, SarahC. Hsu, ChrisC.-S. Hsi- ${ }_{725}$ ung, Arjun Raj, and GerdA. Blobel. Enhancer Reg-726 ulation of Transcriptional Bursting Parameters Re- 727 vealed by Forced Chromatin Looping. Molecular Cell, ${ }_{728}$ 62(2):237-247, April 2016. ISSN 10972765. doi: 729 10.1016/j.molcel.2016.03.007. URL http://linkinghub. 730 elsevier.com/retrieve/pii/S1097276516001854.

[11] Jeehae Park, Javier Estrada, Gemma Johnson, Ben $J_{732}$ Vincent, Chiara Ricci-Tam, Meghan Dj Bragdon, Yeka-7з3 terina Shulgina, Anna Cha, Zeba Wunderlich, Jeremy Gunawardena, and Angela H DePace. Dissecting the ${ }_{735}$ sharp response of a canonical developmental enhancer ${ }_{736}$ reveals multiple sources of cooperativity. eLife, 8:2787,737 June 2019.

[12] Mark Ptashne. A genetic switch: gene control and phage ${ }_{73}$ [lambda]. Cell Press Cambridge, MA, 1986.
13] Thomas Kuhlman, Zhongge Zhang, Milton H Saier, and Terence Hwa. Combinatorial transcriptional control of the lactose operon of escherichia coli. Proceedings of the National Academy of Sciences, 104(14):6043-6048, 2007.

[14] Otto G Berg and Peter H von Hippel. Selection of dna binding sites by regulatory proteins: Statisticalmechanical theory and application to operators and promoters. Journal of molecular biology, 193(4):723-743, 1987.

[15] J. B. Kinney, A. Murugan, C. G. Callan, and E. C. Cox. Using deep sequencing to characterize the biophysical mechanism of a transcriptional regulatory sequence. Proceedings of the National Academy of Sciences, 107(20):9158-9163, May 2010. ISSN 0027-8424, 1091-6490. doi:10.1073/pnas.1004290107. URL http: //www.pnas.org/cgi/doi/10.1073/pnas.1004290107.

[16] Nathan M. Belliveau, Justin B. Kinney, and Rob Phillips. Systematic approach for dissecting the moleclar mechanisms of transcriptional regulation in bacteria. PNAS, page 10, May 2018.

[17] Hernan G Garcia, Alvaro Sanchez, James Q Boedicker, Melisa Osborne, Jeff Gelles, Jane Kondev, and Rob Phillips. Operator sequence alters gene expression independently of transcription factor occupancy in bacteria. Cell reports, 2(1):150-161, 2012.

[18] Petter Hammar, Mats Walldén, David Fange, Fredrik Persson, Özden Baltekin, Gustaf Ullman, Prune Leroy, and Johan Elf. Direct measurement of transcription factor dissociation excludes a simple operator occupancy model for gene regulation. Nature genetics, 46(4):405, 2014.

[19] Talitha L Forcier, Andalus Ayaz, Manraj S Gill, Daniel Jones, Rob Phillips, and Justin B Kinney. Measuring cis-regulatory energetics in living cells using allelic manifolds. Elife, 7:e40618, 2018.

[20] Lacramioara Bintu, Nicolas E Buchler, Hernan G Garcia, Ulrich Gerland, Terence Hwa, Jan Kondev, Thomas Kuhlman, and Rob Phillips. Transcriptional regulation by the numbers: applications. Current Opinion in Genetics \& Development, 15 (2):125-135, April 2005. ISSN 0959437X. doi: 10.1016/j.gde.2005.02.006. URL https://linkinghub. elsevier.com/retrieve/pii/S0959437X05000298.

[21] Lacramioara Bintu, Nicolas E Buchler, Hernan G Garcia, Ulrich Gerland, Terence Hwa, Jan Kondev, and Rob Phillips. Transcriptional regulation by the numbers: models. Current Opinion in Genetics 85 Development, 15(2):116-124, April 2005. ISSN 0959437X. doi: 10.1016/j.gde.2005.02.007. URL https://linkinghub. elsevier.com/retrieve/pii/S0959437X05000304.

[22] Sebastian J Maerkl and Stephen R Quake. A systems approach to measuring the binding energy landscapes of transcription factors. Science, 315(5809):233-237, 2007.

[23] Daniel L Jones, Robert C Brewster, and Rob Phillips. Promoter architecture dictates cell-to-cell variability in gene expression. Science, 346(6216):1533-1536, 2014.

[24] Tamar Friedlander, Roshan Prizak, Clin C. Guet, Nicholas H. Barton, and Gaper Tkaik. Intrinsic limits to gene regulation by global crosstalk. Nature Communications, 7:12307, August 2016. ISSN 2041-1723. doi: 10.1038/ncomms12307. URL http://www. nature.com/ doifinder/10.1038/ncomms12307.

[25] Sarah A. Cepeda-Humerez, Georg Rieckh, and Gaper 
Tkaik. Stochastic Proofreading Mechanism Alleviates804 Crosstalk in Transcriptional Regulation. Physical Re-805 view Letters, 115(24), December 2015. ISSN 0031-9007,806 1079-7114. doi:10.1103/PhysRevLett.115.248101. URL807 https://link.aps.org/doi/10.1103/PhysRevLett. $\quad 808$ 115.248101.

26] Benjamin T Donovan, Anh Huynh, David A Ball, Heta P810 Patel, Michael G Poirier, Daniel R Larson, Matthew L811 Ferguson, and Tineke L Lenstra. Live-cell imaging revealssi2 the interplay between transcription factors, nucleosomes,813 and bursting. The EMBO Journal, 38(12):e100809-18,814 June 2019 .

[27] Javier Estrada, Felix Wong, Angela DePace, and816 Jeremy Gunawardena. Information Integration and817 Energy Expenditure in Gene Regulation. Cell, 166818 (1):234-244, June 2016. ISSN 00928674. doi:819 10.1016/j.cell.2016.06.012. URL http://linkinghub. 820 elsevier.com/retrieve/pii/S0092867416307413.

[28] Jason Gertz, Eric D. Siggia, and Barak A. Cohen.822 Analysis of combinatorial cis-regulation in synthetic823 and genomic promoters. Nature, 457(7226):215-218,824 January 2009. ISSN 0028-0836, 1476-4687. doi:825 10.1038/nature07521. URL http://www.nature.com/826 articles/nature07521.

[29] Gašper Tkačik and Aleksandra M Walczak. Informations28 transmission in genetic regulatory networks: a review.829 Journal of Physics: Condensed Matter, 23(15):153102,8зо 2011.

831

[30] Georg Rieckh and Gašper Tkačik. Noise and information832 transmission in promoters with multiple internal states.833 Biophysical journal, 106(5):1194-1204, 2014.

834

[31] Gašper Tkačik and William Bialek. Information process-835 ing in living systems. Annual Review of Condensed Mat-836 ter Physics, 7:89-117, 2016.

[32] Rob Phillips, Julie Theriot, Jane Kondev, and Hernansзs Garcia. Physical biology of the cell. Garland Science,8з9 2012.

840

[33] L. A. Mirny. Nucleosome-mediated cooperativity be-841 tween transcription factors. Proceedings of the $\mathrm{Na}-842$ tional Academy of Sciences, 107(52):22534-22539, De-843 cember 2010. ISSN 0027-8424, 1091-6490. doi:844 10.1073/pnas.0913805107. URL http://www.pnas.org/845 cgi/doi/10.1073/pnas.0913805107.

[34] Aleksandra M Walczak, Gašper Tkačik, and William847 Bialek. Optimizing information flow in small genetic net-848 works. ii. feed-forward interactions. Physical Review E,849 81(4):041905, 2010.

850

35] Jean-Pierre Changeux. Allostery and the monod-wyman-851 changeux model after 50 years. Annual review of bio-852 physics, 41:103-133, 2012.

[36] Daniel R Larson, Christoph Fritzsch, Liang Sun, Xiuhau854 Meng, David S Lawrence, and Robert H Singer. Direct 855 observation of frequency modulated transcription in sin-856 gle cells using light activation. eLife, 2:e00750, 2013.

[37] Adrien Senecal, Brian Munsky, Florence Proux, Nathalies58 Ly, FlorianeE. Braye, Christophe Zimmer, Florian859 Mueller, and Xavier Darzacq. Transcription Fac-860 tors Modulate c-Fos Transcriptional Bursts. Cell Re-861 ports, 8(1):75-83, July 2014. ISSN 22111247. doi:862 10.1016/j.celrep.2014.05.053. URL http://linkinghub. 863 elsevier.com/retrieve/pii/S2211124714004471. 864

[38] Benjamin Zoller, Shawn C Little, and Thomas Gregor.865 Diverse Spatial Expression Patterns Emerge from Unified 866 Kinetics of Transcriptional Bursting. Cell, 175(3):835-867 847.e25, October 2018.

[39] Alvaro Sanchez and Jané Kondev. Transcriptional control of noise in gene expression. Proceedings of the $\mathrm{Na}$ tional Academy of Sciences, 105(13):5081-5086, April 2008.

[40] Ioannis Lestas, Johan Paulsson, Nicholas E Ross, and Glenn Vinnicombe. Noise in Gene Regulatory Networks. Automatic Control, IEEE Transactions on, 53:189-200, 2008.

[41] Aleksandra M. Walczak, Andrew Mugler, and Chris H. WIggins. Analytic methods for modeling stochastic regulatory networks. Methods in molecular biology (Clifton, N.J.), 880(Chapter 13):273-322, 2012.

[42] Daniel T Gillespie. Stochastic simulation of chemical kinetics. Annual Review of Physical Chemistry, 58:35-55, 2007.

[43] Tatsuya Morisaki, Waltraud G Müller, Nicole Golob, Davide Mazza, and James G McNally. Single-molecule analysis of transcription factor binding at transcription sites in live cells. Nature Communications, 5(1):4456, July 2014.

[44] Daniel Zenklusen, Daniel R Larson, and Robert H Singer. Single-RNA counting reveals alternative modes of gene expression in yeast. Nature Structural \& Molecular Biology, 15(12):1263-1271, December 2008.

[45] David M Suter, Nacho Molina, David Gatfield, Kim Schneider, Ueli Schibler, and Felix Naef. Mammalian genes are transcribed with widely different bursting kinetics. Science, 332(6028):472-474, April 2011.

[46] B. Zoller, D. Nicolas, N. Molina, and F. Naef. Structure of silent transcription intervals and noise characteristics of mammalian genes. Molecular Systems Biology, 11(7):823-823, July 2015. ISSN 1744-4292. doi: 10.15252/msb.20156257. URL http://msb.embopress. org/cgi/doi/10.15252/msb. 20156257.

[47] John J. Hopfield. Kinetic proofreading: a new mechanism for reducing errors in biosynthetic processes requiring high specificity. Proceedings of the National Academy of Sciences, 71(10):4135-4139, 1974.

[48] David N Arnosti and Meghana M Kulkarni. Transcriptional enhancers: Intelligent enhanceosomes or flexible billboards? Journal of cellular biochemistry, 94(5):890898, 2005.

[49] Johan Paulsson. Summing up the noise in gene networks. Nature, 427(6973):415-418, January 2004.

[50] Gašper Tkačik, Thomas Gregor, and William Bialek. The role of input noise in transcriptional regulation. PloS one, 3(7), 2008.

[51] Hongtao Chen, Michal Levo, Lev Barinov, Miki Fujioka, James B Jaynes, and Thomas Gregor. Dynamic interplay between enhancer-promoter topology and gene activity. Nature genetics, 50(9):1296-1303, 2018.

[52] Won-Ki Cho, Jan-Hendrik Spille, Micca Hecht, Choongman Lee, Charles Li, Valentin Grube, and Ibrahim I Cisse. Mediator and RNA polymerase II clusters associate in transcription-dependent condensates. Science, 361(6400):412-415, July 2018.

[53] Benjamin R Sabari, Alessandra Dall'Agnese, Ann Boija, Isaac A Klein, Eliot L Coffey, Krishna Shrinivas, Brian J Abraham, Nancy M Hannett, Alicia V Zamudio, John C Manteiga, Charles H Li, Yang E Guo, Daniel S Day, Jurian Schuijers, Eliza Vasile, Sohail Malik, Denes Hnisz, Tong Ihn Lee, Ibrahim I Cisse, Robert G Roeder, Phillip A Sharp, Arup K Chakraborty, and Richard A 
Young. Coactivator condensation at super-enhancers896 links phase separation and gene control. Science, 361897 (6400), July 2018.

[54] Gang Ren, Wenfei Jin, Kairong Cui, Joseph Rodrigez,899 Gangqing Hu, Zhiying Zhang, Daniel R Larson, and Kejigoo Zhao. CTCF-Mediated Enhancer-Promoter Interaction901 Is a Critical Regulator of Cell-to-Cell Variation of Gene9o2 Expression. Molecular Cell, 67(6):1049-1058.e6, Septem-903 ber 2017.

[55] Denes Hnisz, Krishna Shrinivas, Richard A. Young,905 Arup K. Chakraborty, and Phillip A. Sharp. A Phase9o6 Separation Model for Transcriptional Control. Cell,907 169(1):13-23, March 2017. ISSN 00928674. doi:908 10.1016/j.cell.2017.02.007. URL http://linkinghub.909 elsevier.com/retrieve/pii/S009286741730185X.

910

[56] William Bialek, Thomas Gregor, and Gašper Tkačik. Ac-911 tion at a distance in transcriptional regulation. arXivis preprint arXiv:1912.08579, 2019.

[57] Tamar Friedlander, Roshan Prizak, Nicholas H. Barton,914 and Gaper Tkaik. Evolution of new regulatory functions915 on biophysically realistic fitness landscapes. Nature Com-916 munications, 8(1), December 2017. ISSN 2041-1723. doi:917 10.1038/s41467-017-00238-8. URL http://www . nature . 918 com/articles/s41467-017-00238-8.

[58] Rene C Adam, Hanseul Yang, Shira Rockowitz, Saman-920 tha B Larsen, Maria Nikolova, Daniel S Oristian, Lisa921 Polak, Meelis Kadaja, Amma Asare, Deyou Zheng, and Elaine Fuchs. Pioneer factors govern super-enhancer dy- namics in stem cell plasticity and lineage choice. Nature, 521(7552):366-370, May 2015.

[59] Sandy L Klemm, Zohar Shipony, and William J Greenleaf. Chromatin accessibility and the regulatory epigenome. Nature Reviews Genetics, 20(4):207-220, April 2019.

[60] Kevin Struhl. Transcriptional noise and the fidelity of initiation by RNA polymerase II. Nature Structural \& Molecular Biology, 14(2):103-105, February 2007.

[61] Andreas H Ehrensberger, Gavin P Kelly, and Jesper Q Svejstrup. Mechanistic interpretation of promoterproximal peaks and RNAPII density maps. Cell, 154(4): 713-715, August 2013.

[62] Mark D Biggin. Animal transcription networks as highly connected, quantitative continua. Developmental cell, 21 (4):611-626, October 2011.

[63] Howard C Berg and Edward M Purcell. Physics of chemoreception. Biophysical journal, 20(2):193-219, 1977.

[64] William Bialek and Sima Setayeshgar. Physical limits to biochemical signaling. Proceedings of the National Academy of Sciences, 102(29):10040-10045, 2005.

[65] Kazunari Kaizu, Wiet De Ronde, Joris Paijmans, Koichi Takahashi, Filipe Tostevin, and Pieter Rein Ten Wolde. The berg-purcell limit revisited. Biophysical journal, 106 (4):976-985, 2014. 